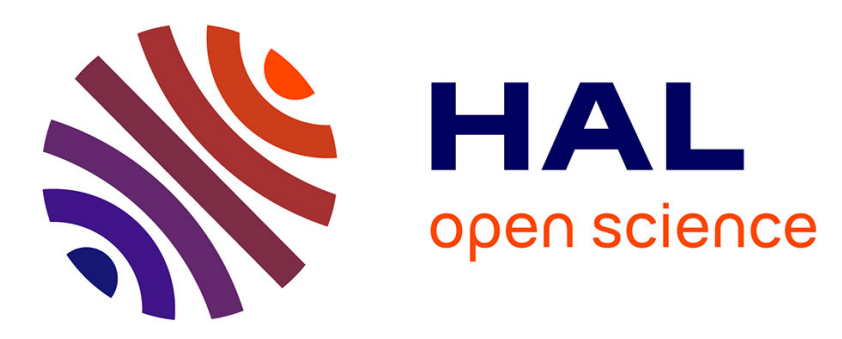

\title{
Extending multifractal analysis to negative regularity: p-exponents and p-leaders
}

\author{
Roberto Leonarduzzi, Herwig Wendt, Stéphane Jaffard, Stéphane Roux, \\ Maria Torres, Patrice Abry
}

\section{- To cite this version:}

Roberto Leonarduzzi, Herwig Wendt, Stéphane Jaffard, Stéphane Roux, Maria Torres, et al.. Extending multifractal analysis to negative regularity: p-exponents and p-leaders. IEEE International Conference on Acoustics, Speech, and Signal Processing - ICASSP 2014, May 2014, Florence, Italy. pp. 305-309. hal-01147322

\section{HAL Id: hal-01147322 \\ https://hal.science/hal-01147322}

Submitted on 30 Apr 2015

HAL is a multi-disciplinary open access archive for the deposit and dissemination of scientific research documents, whether they are published or not. The documents may come from teaching and research institutions in France or abroad, or from public or private research centers.
L'archive ouverte pluridisciplinaire HAL, est destinée au dépôt et à la diffusion de documents scientifiques de niveau recherche, publiés ou non, émanant des établissements d'enseignement et de recherche français ou étrangers, des laboratoires publics ou privés. 


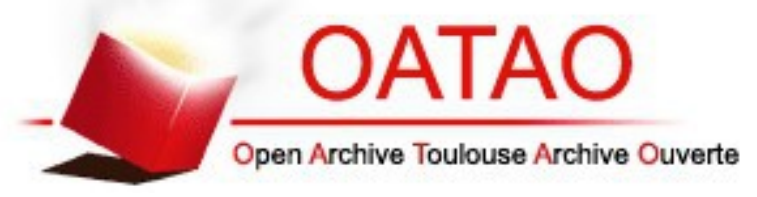

\section{Open Archive TOULOUSE Archive Ouverte (OATAO)}

OATAO is an open access repository that collects the work of Toulouse researchers and makes it freely available over the web where possible.

This is an author-deposited version published in : http://oatao.univ-toulouse.fr/ Eprints ID : 12911

To link to this article : DOI :10.1109/ICASSP.2014.6853607 URL : http://dx.doi.org/10.1109/ICASSP.2014.6853607

To cite this version : Leonarduzzi, Roberto and Wendt, Herwig and Jaffard, Stéphane and Roux, Stéphane and Torres, Maria and Abry, Patrice Extending multifractal analysis to negative regularity: $p$ exponents and p-leaders. (2014) In: IEEE International Conference on Acoustics, Speech, and Signal Processing - ICASSP 2014, 5 May 2014 - 9 May 2014 (Florence, Italy).

Any correspondance concerning this service should be sent to the repository administrator: staff-oatao@,listes-diff.inp-toulouse.fr 


\title{
EXTENDING MULTIFRACTAL ANALYSIS TO NEGATIVE REGULARITY: P-EXPONENTS AND P-LEADERS
}

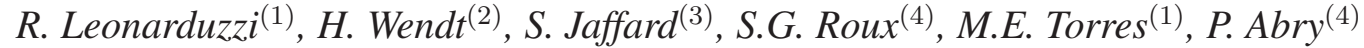 \\ (1) CONICET, Universidad Nacional de Entre Rios, Argentina, rleonarduzzi@bioingenieria.edu.ar, \\ (2) IRIT - ENSEEIHT, CNRS, Toulouse, France, herwig.wendteirit.fr \\ (3) LAMA, Math. Dept., Paris Est Univ., Créteil, France, jaffardeu-pec.fr \\ (4) SISYPhe, Physics Dept., ENS Lyon, CNRS, Lyon, France, patrice.abryeens-1yon.fr \\ Work supported by ANR BLANC 2011 AMATIS BS0101102 and CNRS-CONICET grant 2010112432
}

\begin{abstract}
Scale invariance is a widely used concept to analyze real-world data from many different applications and multifractal analysis has become the standard corresponding signal processing tool. It characterizes data by describing globally and geometrically the fluctuations of local regularity, usually measured by means of the Hölder exponent. A major limitation of the current procedure is that it applies only to locally bounded functions or signals, i.e., to signals with positive regularity. The present contribution proposes to characterize local regularity with a new quantity, the p-exponent, that permits negative regularity in data, a widely observed property in real-world data. Relations to Hölder exponents are detailed and a corresponding p-leader multifractal formalism is devised and shown at work on synthetic multifractal processes, representative of a class of models often used in applications. We formulate a conjecture regarding the equivalence between Hölder and p-exponents for a subclass of processes. Even when Hölder and p-exponents coincide, the p-leader formalism is shown to achieve better estimation performance.
\end{abstract}

Index Terms - scale invariance, multifractal analysis, negative local regularity exponent, wavelet Leaders, estimation performance

\section{CONTEXT, GOALS AND CONTRIBUTIONS}

Scale invariance and Multifractal Analysis. Multifractal analysis $[1,2]$ has recently matured to a standard signal processing tool that provides a fine characterization of the scaling properties of time series (cf. e.g., $[1,2,3])$. In essence, multifractal analysis relies on the notion of local regularity, theoretically grounded in the definition of the Hölder exponent $h(t)[1,2]$ : The closer $h(t)$ is to 0 , the more irregular a signal $X$ is at time position $t$; conversely, the larger $h(t)$, the more regular $X$ at $t$. Multifractal analysis does, however, not aim at characterizing $X$ via the fluctuations of its local regularity along time, i.e., via the function $h(t)$. Instead, it provides practitioners with a characterization of $X$ via the multifractal spectrum $\mathcal{D}(h)$, consisting of a global geometrical description of the fluctuations of $h(t)$. The practical estimation of $\mathcal{D}(h)$ is achieved through a procedure referred to as a multifractal formalism. It relies on the use of multiresolution quantities $T_{X}(a, t)$, i.e., of quantities that measure the behavior of $X$ around $t$ at scale $a$, such as wavelet coefficients. The asymptotic behavior of the time average of the moments of order $q$ of $T_{X}(a, t)$ in the limit of fine scales theoretically defines the scaling function

$$
\zeta(q)=\lim _{a \rightarrow 0} \log \left(1 / n_{a} \sum_{k}\left|T_{X}(a, k)\right|^{q}\right) / \log a,
$$

whose Legendre transform provides an estimate of $\mathcal{D}(h)$ in form of an upper bound (cf. Section 2 and $[1,2])$.

The definition of $\zeta(q)$ above in essence amounts to assuming a scaling behavior with respect to the analysis scale $a$,

$$
1 / n_{a} \sum_{k}\left|T_{X}(a, k)\right|^{q} \simeq c_{q} a^{\zeta(q)}, a \rightarrow 0,
$$

connecting multifractal analysis as an analysis tool and scale invariance as a modeling paradigm. In practice, the asymptotic power law above is assumed to hold over a finite yet large range of scales $a_{m} \leq a \leq a_{M}$, with $a_{M} / a_{m} \gg 1$, thus enabling the estimation of the scaling exponents $\zeta(q)$ and further of $\mathcal{D}(h)$.

Multifractal analysis has been successfully used as a tool to analyze, describe, model and classify temporal dynamics of signals in numerous real-world applications of very different types, including hydrodynamic turbulence [4], biomedical data and body rhythms $[5,6]$, geophysical data $[7,8]$, fMRI $[9,10]$, finance [11] and image textures [12]. For reviews and examples, see e.g., [13, 14].

Related works: recent developments and open issues. Multifractal analysis has received significant research efforts at both the theoretical and application levels. Most of the recent developments aiming at improving multifractal analysis, either in theoretical foundations or in applied schemes, focus on exploring the benefits of varying the multiresolution quantities $T_{X}(a, t)$ serving as the basic analysis unit. Increments, oscillations, wavelet coefficients, continuous wavelet transform modulus maxima $[15,16]$ and more recently wavelet leaders [3] were used in the vast majority of cases. These developments were mainly concerned with parameter estimation performance and robustness (cf. [3, 17] and references therein), or, more recently, with analysis refinement (e.g., detection of oscillating singular behaviors in data [18].) Along another line, procedures avoiding the Legendre transform based formalism (which yields the convex hull of $\mathcal{D}(h)$ only) have been investigated. Notably, large deviation principle based approaches were studied, aiming at the estimation of non convex multifractal spectra (cf. e.g., $[2,19])$.

However, none of these developments reflected two fundamental facts. First, by definition, the Hölder exponent is positive, $h(t) \geq 0$. Consequently, its use as a measure of local regularity induces a major a limitation for the application of multifractal analysis to real-world data: It permits the analysis of the fluctuations of local regularity for locally bounded functions only. Second, the choice of a particular multiresolution quantity $T_{X}(a, t)$ is tied to the way local regularity is measured and can therefore imply a change in the definition of the exponent used to measure local regularity. Often, in applications, real-world data are well modeled by stochastic processes 
whose sample paths do not constitute locally bounded functions, this is the case e.g., for fractional Gaussian noise or multifractal random walks [20], frequently used to model Heart Rate Variability [6], fMRI fluctuations $[9,10]$ or Mandelbrot's multiplicative cascades, used to model, e.g., dissipation in hydrodynamic turbulence [4], rainfalls [7], cloud textures [15], to list but a few examples. In these situations, the Hölder exponent is of limited practical use, and so are the associated multiresolution quantities.

Recently, an alternative measure of regularity, the p-exponent $h_{p}(t)$, has been introduced theoretically in the context of multifractal analysis [21]. One of its potential advantages is that it enables to weaken the positive regularity and boundedness requirement and admits a certain range of negative exponents. It has, to the best of our knowledge, not been used in applications and remains poorly studied in terms of practical applicability and estimation performance.

Goals, contributions and outline. In this context, the goals of the present contribution are three-fold: First, it aims at defining the p-exponent, reviewing the key differences with the Hölder exponent, and devising the corresponding multiresolution quantities and multifractal formalism (cf. Section 2). Second, the principles and limitations of the p-exponent based multifractal formalism are illustrated on sample paths of a stochastic process chosen as a representative model for scale invariance in applications (cf. Section 3). Third, equality between Hölder and p-exponents is conjectured for a class of model processes. In the case of coinciding exponents, it is shown numerically that the p-leader formalism achieves better estimation performance than the wavelet leader formalism, now considered as a benchmark for practical multifractal analysis (cf. Section 4).

\section{LOCAL REGULARITY, MULTIFRACTAL ANALYSIS}

\subsection{Hölder regularity}

Wavelet coefficients. Let $\{X(t)\}_{t \in \mathbb{R}}$ denote the signal to be analyzed. Let $\psi$ denote the mother wavelet, characterized by its uniform regularity index and number of vanishing moments $N_{\psi}$, a strictly positive integer defined as: $\psi \in C^{N_{\psi}-1}$ and $\forall n=0, \ldots, N_{\psi}-1, \int_{\mathbb{R}} t^{k} \psi(t) d t \equiv 0$ and $\int_{\mathbb{R}} t^{N_{\psi}} \psi(t) d t \neq 0$. Let $\left\{\psi_{j, k}(t)=2^{-j / 2} \psi\left(2^{-j} t-k\right)\right\}_{(j, k) \in \mathbb{N}^{2}}$ denote the collection of dilated and translated templates of $\psi$ that form an orthonormal basis of $\mathcal{L}^{2}(\mathbb{R})$. The ( $L^{1}$-normalized) discrete wavelet transform coefficients $d_{X}(j, k)$ of $X$ are defined as $d_{X}(j, k)=2^{-j / 2}\left\langle\psi_{j, k} \mid X\right\rangle$. For a detailed introduction to wavelet transforms, readers are referred to, e.g., [22] . Early formulations of multifractal analysis were based on the structure functions of wavelet coefficients, of order $q \geq 0$,

$$
\eta(q)=1 / n_{j} \sum_{k}\left|d_{X}(j, k)\right|^{q},
$$

but have been shown to suffer from poor practical performance (cf. e.g., $[16,23,1,3])$, this is thus not further discussed here.

Hölder exponent. Assume that $\{X(t)\}_{t \in \mathbb{R}}$ consists of a locally bounded function (or sample path of a stochastic process), i.e., belongs to $L^{\infty}(\mathbb{R})$. Then $X$ is said to belong to $C_{\alpha}(t)$ at time position $t \in \mathbb{R}$, with $\alpha \geq 0$, if there exist a constant $C>0$ and a polynomial $P_{t}$ satisfying $\operatorname{Deg}\left(P_{t}\right)<\alpha$ such that, in a neighborhood of $t$ :

$$
\left|X(t+a)-P_{t}(t+a)\right| \leq C|a|^{\alpha},|a| \rightarrow 0 .
$$

The Hölder exponent of $X$ at $t$ is defined as:

$$
h(t)=\sup \left\{\alpha: X \in C^{\alpha}(t)\right\} \geq 0 .
$$

It characterizes the local regularity of $X$ at $t$ in the sense that the larger (smaller) $h(t)$, the smoother (rougher) $X$ around $t$. The simplest example of a singularity at datum $t_{0}$ whose Hölder exponent is $h$ (when $h$ is not a even integer) is supplied by the cusp-type function $X(t)=X\left(t_{0}\right)+B\left|t-t_{0}\right|^{h}$.

Multifractal spectrum and wavelet leaders. Though deeply rooted in the notions of local regularity and Hölder exponents, multifractal analysis does not aim at characterizing $X$ through regularity as a function of time $h(t)$. Instead, it provides practitioners with a global and geometrical description of the fluctuations along time of the Hölder exponent $h$, in terms of the multifractal spectrum $\mathcal{D}(h)$. Technically, $\mathcal{D}(h)$ is given by the Hausdorff dimensions of the sets of points where the Hölder exponent takes the value $h$ (for details, the interested reader is referred to $[23,2,1]$.)

The practical measurement of $\mathcal{D}(h)$ relies on a procedure referred to as the multifractal formalism: It requires the definition of multiresolution quantities, the wavelet leaders $L_{X}(j, k)$, defined as the local supremum of wavelet coefficients taken within a neighborhood over all finer scales $[1,3]$ :

$$
\begin{gathered}
L_{X}(j, k)=\sup _{\lambda^{\prime} \subset 3 \lambda_{j, k}}\left|d_{X}\left(\lambda^{\prime}\right)\right|, \text { where } \\
\lambda_{j, k}=\left[k 2^{j},(k+1) 2^{j}\right) \text { and } 3 \lambda_{j, k}=\bigcup_{m\{-1,0,1\}} \lambda_{j, k+m} .
\end{gathered}
$$

The $L_{X}(j, k)$ match the Hölder exponent in the sense that for $t=2^{j} k, L_{X}(j, k) \sim C 2^{j h(t)}$ as $2^{j} \rightarrow 0$. This implies that $\frac{1}{n_{j}} \sum_{k=1}^{n_{j}} L_{X}(j, k)^{q} \sim c_{q} 2^{j \zeta(q)}$ as $2^{j} \rightarrow 0$ as in (1) and that the Legendre transform of $\zeta(q)$ yields an (upper-bound) estimate of the multifractal spectrum, $\mathcal{L}(h)=\min _{q}(1+q h-\zeta(q)) \geq \mathcal{D}(h)$.

Limitation. The key limitation in the practical use of the Hölder based multifractal formalism resides in its applicability to locally bounded functions only. This requirement implies the equivalent condition that the minimum regularity $h_{\mathrm{m}}=\inf _{t} h(t)$ of the data is non-negative, which can be practically checked prior to application of the multifractal formalism by using the wavelet coefficient based estimate

$$
h \mathrm{~m}=\lim _{2^{j} \rightarrow 0} \log _{2} \sup _{k}\left|d_{X}(j, k)\right| / \log _{2} 2^{j} .
$$

\section{2. p-exponent regularity}

p-exponents. We extend local regularity measures to negative values by replacing the bounded function requirement by the condition that $X(t)$ locally belongs to $L^{p}(\mathbb{R})$ for $p \geq 1: X$ is said to belong to $T_{\alpha}^{p}(t)$ with $\alpha>-1 / p$ at time $t$ if there exist $C, R>0$ and a polynomial $P_{t}$ (of degree $N$ less than or equal to $\alpha$ ) such that $\forall a \leq R$

$$
T_{X}^{(p)}(a, t)=\left(\frac{1}{a} \int_{t-a / 2}^{t+a / 2}\left|X(u)-P_{t, N}(u-t)\right|^{p} d u\right)^{1 / p} \leq C a^{\alpha} .
$$

The p-exponent $h_{p}(t)$ of $X$ at $t$ is defined as [21]:

$$
h_{p}(t)=\sup \left\{\alpha: X \in T_{\alpha}^{p}(t)\right\}
$$

and constitutes a natural substitute for the Hölder exponent when dealing with functions which are not bounded but locally belong to $L^{p}$ and admits negative local regularity exponents $h_{p}>-1 / p$. Obviously, the Hölder exponent coincides with the p-exponent for $p=+\infty: h(t) \equiv h_{\infty}(t)$; furthermore, $h_{p^{\prime}} \leq h_{p}$ if $p^{\prime} \geq p[21,24]$. p-multifractal spectrum and p-leaders. Mimicking the definition of $\mathcal{D}(h)$, mutatis mutandis, the p-multifractal spectrum $\mathcal{D}^{(p)}\left(h_{p}\right)$ is defined as the Hausdorff dimensions of the sets of points where the p-exponent takes the value $h_{p}$. 
The practical measurement of $\mathcal{D}^{(p)}(h)$ requires the definition of new multiresolution quantities, the $p$-leaders $[25,26,21]$ :

$$
L^{(p)}(j, k)=\left(2^{j} \sum_{\lambda^{\prime} \subset 3 \lambda_{j}}\left|d_{\lambda^{\prime}}\right|^{p} 2^{-j^{\prime}}\right)^{1 / p}
$$

that match p-exponent local regularity in the sense that

$$
\text { for } t=2^{j} k, L^{(p)}(j, k) \sim C 2^{j h_{p}(t)}, 2^{j} \rightarrow 0 .
$$

The p-scaling function is defined similarly as in (1):

$$
S(p, q, j)=1 / n_{j} \sum_{k=1}^{n_{j}} L^{(p)}(j, k)^{q} \sim C_{q}^{(p)} 2^{j \zeta_{p}(q)}, 2^{j} \rightarrow 0 ;
$$

and the Legendre transform of $\zeta_{p}(q)$ yields an (upper-bound) estimate of $\mathcal{D}^{(p)}\left(h_{p}\right)$,

$$
\mathcal{L}^{(p)}\left(h_{p}\right)=\min _{q}\left(1+q h_{p}-\zeta_{p}(q)\right) \geq \mathcal{D}^{(p)}\left(h_{p}\right) .
$$

The demonstration of that results follows from the proof given in [27], which is generic for any well-behaved multiresolution quantity.

In practice, $X \in L^{p}(\mathbb{R})$ must be verified prior to application of the p-leader multifractal formalism, by checking that $\eta(p) \geq 0[21$, $25,26]$, or equivalently that its Legendre transform $\mathcal{L}(h)$ satisfies

$$
\mathcal{L}(h) \leq 1+p h
$$

Cumulants and estimation. Following the original intuition in [28], a polynomial expansion $\zeta_{p}(q)=\sum_{m \geq 1} c_{m}^{(p)} q^{m} / m$ ! enables a simplified analysis of $\zeta_{p}(q)$ through the expansion coefficients $\left\{c_{l}^{(p)}, c_{2}^{(p)}, c_{3}^{(p)}, \ldots\right\}$. Reproducing the calculations in [28] for $\mathrm{p}$ leaders immediately yields that these coefficients are directly related to the dependence with respect to scales $2^{j}$ of the cumulants of order $m$, denoted by $C_{m}^{(p)}(j)$, of the logarithm of the p-leaders:

$$
C_{m}^{(p)}(j)=\mathrm{Cum}_{m} \ln L^{(p)}(j, k)=c_{m}^{(0, p)}+c_{m}^{(p)} \ln 2^{j} .
$$

This scaling behavior is used for the practical estimation of the coefficients $c_{m}^{(p)}$ by linear regressions of the estimates of $C_{m}^{(p)}(j)$ against $\ln 2^{j}$, for $j \in\left[j_{1}, j_{2}\right]$. In view of (11), the $\zeta_{p}(q)$ are also estimated by linear regressions of $\log _{2} S(p, q, j)$ versus $\log _{2} 2^{j}=j$. These estimation procedures, implemented by ourselves, are used to obtain the results produced in Sections 3 and 4.

Hölder versus p-exponents. In general, p-exponents do not coincide for different $p$ s nor with Hölder exponent (see [24] for a theoretical discussion); thus the associated spectrum yields a novel and versatile collection of characterizations of local regularity fluctuations, further illustrated and studied in Sections 3 and 4.

\section{NEGATIVE REGULARITY}

Multifractal random walk. The differences and potential benefits in using p-exponents and p-leaders for the characterization of local regularity fluctuations are now illustrated on pedagogical examples, containing negative p-exponents. They are based on fractionally differentiated realizations of multifractal random walk (MRW) $[20,29]$, a popular and representative member of the class of multiplicative cascade based multifractal processes. This class constitutes one of the most prominently used class of multifractal models for applications. MRW is a non Gaussian process with stationary
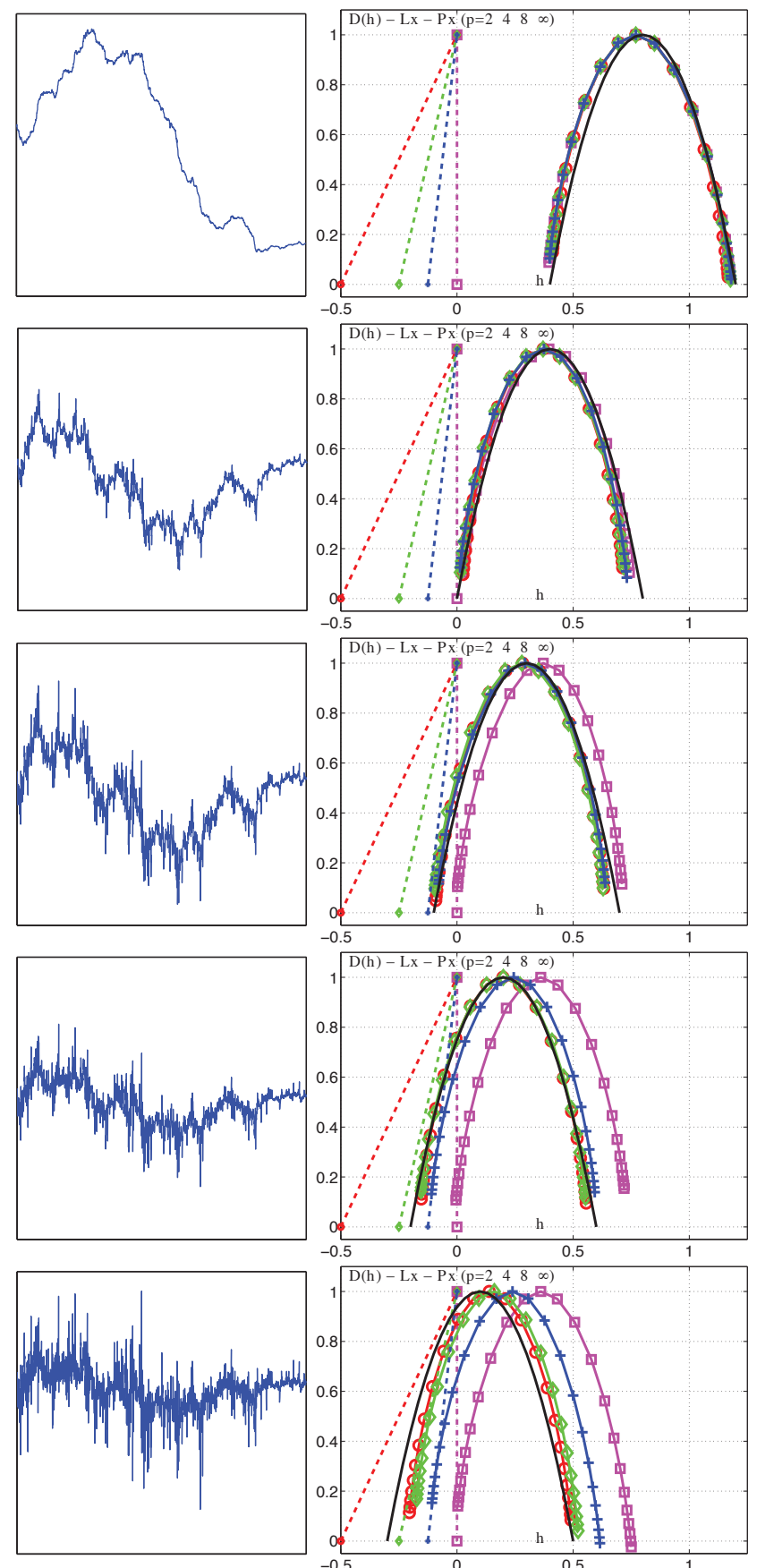

Fig. 1. Left column: A single realization of fractionally differen-

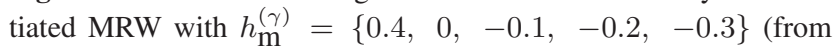
top to bottom, respectively). Right column: The theoretical multifractal spectra (black solid) and Legendre spectra (mean over 50 realizations, $N=2^{19}, N_{\psi}=2, j_{1}=10$ ) corresponding with the functions in the left column for $p=\{2,4,8, \infty\}$ (solid lines; red circle, green diamond, blue cross and magenta square); the dashed line segments indicate the theoretical bound $1+h p$ for the spectrum of any $L^{p}$ function (in respective colors for the different $p$ ). 
increments and its multifractal properties mimic those of the celebrated multiplicative log-normal cascades of Mandelbrot. It is defined as $X(k)=\sum_{k=1}^{n} G_{H}(k) e^{\omega(k)}$, where $G_{H}(k)$ are the increments of fractional Brownian motion with parameter $H$, and $\omega$ is a Gaussian random process, independent of $G_{H}$ and with non trivial autocovariance $\operatorname{Cov}\left[\omega\left(k_{1}\right), \omega\left(k_{2}\right)\right]=c_{2} \ln \left(\frac{L}{\left|k_{1}-k_{2}\right|+1}\right)$ when $\left|k_{1}-k_{2}\right|<L$ and 0 otherwise. MRW has scaling properties as in (1) for $q \in\left[-\sqrt{2 / c_{2}}, \sqrt{2 / c_{2}}\right]$, with $\zeta(q)=\left(H+c_{2}\right) q-c_{2} q^{2} / 2$ and its multifractal spectrum is given by $\mathcal{D}^{(\infty)}(h)=1+\left(h-c_{1}\right)^{2} /\left(2 c_{2}\right)$ (with $c_{1}=H+c_{2}$ and $h \mathrm{~m}=c_{1}-\sqrt{-2 c_{2}}$ ).

Hölder versus p-exponents. Regarding p-exponent analysis, we form the following conjecture, strongly backed up by the numerical simulations reported below, that will be proven in [24]:

Conjecture 1 The p-exponents of multiplicative cascade based multifractal processes $X \in L^{p_{0}}$ coincide for all $p \in\left[1, p_{0}\right]$, hence $\mathcal{D}^{(p)}(h)=\mathcal{D}^{\left(p_{0}\right)}(h)$ for all $p \in\left[1, p_{0}\right]$.

Notably, this conjecture implies that when $h \mathrm{~m} \geq 0$, the Hölder and the p-exponents coincide $\forall p \geq 1$ and thus that the Hölder and the p-exponent based multifractal spectra coincide for MRW, and $\forall p \geq$ 1: $\mathcal{D}^{(p)}(h)=\mathcal{D}^{(\infty)}(h)=\mathcal{D}(h)$.

Numerical experiments. We fix $c_{1}=0.8$ and $c_{2}=-0.08$ and use fractional differentiation of order $\gamma$ to control the minimal regularity and function class embedding of the sample paths, $X^{(\gamma)}=\mathcal{F}^{-1}\left[(1 \omega)^{\gamma} \mathcal{F}[X]\right]$, where $\mathcal{F}$ stands for the discrete Fourier

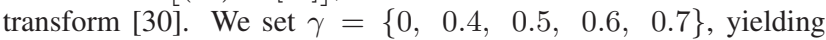
$h_{\mathrm{m}}^{(\gamma)}=h_{\mathrm{m}}-\gamma=\{0.4,0,-0.1,-0.2,-0.3\}, c_{1}^{(\gamma)}=c_{1}-\gamma$, $c_{m}^{(\gamma)}=c_{m}$ for $m \geq 2$ and $p_{0}=\{+\infty,+\infty, 10,5,2.5\}$, where $p_{0}$ denotes the value for $p$ such that $X^{(\gamma)} \in L^{p_{0}}$ and $\forall \varepsilon>0$ : $X^{(\gamma)} \notin L^{p_{0}+\varepsilon}$. We fix $N=2^{19}$. Examples of sample paths are plotted in Fig. 1 (left column) and illustrate the potential of processes with negative p-exponents as models for applications, offering a continuum of moderately to strongly irregular sample paths. The corresponding multifractal spectra and estimates (as detailed in Section 2) for $p=\{2,4,8, \infty\}$ (mean over 50 realizations) are plotted in Fig. 1 (right column), together with the theoretical limits $\mathcal{D}^{(p)}\left(h_{p}\right) \leq 1+p h_{p}$ for the multifractal p-spectra of $L^{p}$ functions. Quality of estimation. First, we observe that in consistency with Conjecture 1, the results obtained with the wavelet leader and pleader formalisms coincide one with another for any of the values of $p$ considered as long as $h_{\mathrm{m}}^{(\gamma)} \geq 0\left(p_{0}=+\infty\right)$, and the formalisms, for any $p$, provide excellent estimates of the multifractal spectrum $\mathcal{D}(h)$ (Fig. 1, row 1-2). Second, as soon as $h_{\mathrm{m}}^{(\gamma)}<0\left(p_{0}<\infty\right)$, the wavelet leader based formalism fails to correctly estimate the spectrum $\mathcal{D}^{\left(p_{0}\right)}(h)$ since the theoretical (bounded function) prerequisite for its application is violated, while the p-leaders estimates $\mathcal{L}^{(p)}(h)$ correctly recover the multifractal spectrum $\mathcal{D}^{\left(p_{0}\right)}(h)$ for values of $p$ such that $p \leq p_{0}$ (Fig. 1, row 3-5). Once this condition for the function to be in $L^{p}$ is violated, the p-leader formalism provides biased estimates $\mathcal{L}^{(p)}(h)$ which are bound to be at best tangent to $1+p h$ (Fig. 1, row 3-5). For example, in row $4, h_{\mathrm{m}}^{(\gamma)}=-0.2$ and estimations $\mathcal{L}^{(p)}$ are found to correctly estimate the theoretical spectrum $\mathcal{D}$ only for $p=2,4<p_{0}=5$, estimates for $p=\{8, \infty\} \geq p_{0}$ are found to differ significantly from the expected spectrum. In row 5 , $h_{\mathrm{m}}^{(\gamma)}=-0.4$ and $\mathcal{L}^{(p)}$ estimates are correct for $p=2<p_{0}=2.5$ only, while $\mathcal{L}^{(p)}$ obtained with $p=\{4,8, \infty\}>p_{0}$ are clearly biased and constrained by the $1+p h$ limits. These results are consistent with the theory outlined in Section 2 and demonstrate the practical effectiveness of the $\mathrm{p}$-leader multifractal formalism in measuring negative values in local regularity.

\section{ESTIMATION PERFORMANCE}

The performance of the wavelet leader and p-leader multifractal formalisms for estimating the $\log$-cumulants $c_{m}, m=1, \ldots, 4$ are compared for 500 realizations from Monte Carlo simulations performed on MRW with process parameters as in Section 3 and $\gamma=0$, hence with $h_{m}>0$ and Hölder and p-exponents and the corresponding spectra coinciding $\forall p \geq 1$. A Daubechies' wavelet with $N_{\psi}=2$ is used, linear regressions are performed over the range $\left(j_{1}, j_{2}\right)=$ $(3,18)$. In Fig. 2, estimation performance are summarized as a function of $p$ in terms of bias $b(\hat{\theta})=\hat{\mathbb{E}}[\hat{\theta}]-\theta$ (blue dashed lines with circles), standard deviations $s(\hat{\theta})=\sqrt{\hat{\mathbb{E}}\left[\hat{\theta}^{2}\right]-\hat{\mathbb{E}}[\hat{\theta}]^{2}}$ (red dashed lines with crosses) and root mean squared errors (rmse) $r(\hat{\theta})=$ $\sqrt{b(\hat{\theta})^{2}+s(\hat{\theta})^{2}}$ (black solid lines with squares).

It is observed that, for $c_{m}, m \geq 2$, variances systematically decrease when $p$ decreases and also that biases decrease or remain constant when $p$ decreases, thus rmse is systematically decreased when $p$ decreases towards 1 . For $c_{1}$, variances appear to decrease only slightly with $p$ while biases tend to re-increase when $p \rightarrow 1$, thus yielding a minimum with an optimal trade-off $p$ around 2 . In overall, it is thus observed that, systematically, when $p$ is decreased, the p-leader based estimation outperforms the wavelet leader based one, currently considered to achieve benchmark performance. Also, it provides practitioners with the possibility of tuning the parameter $p$ to data and applications.

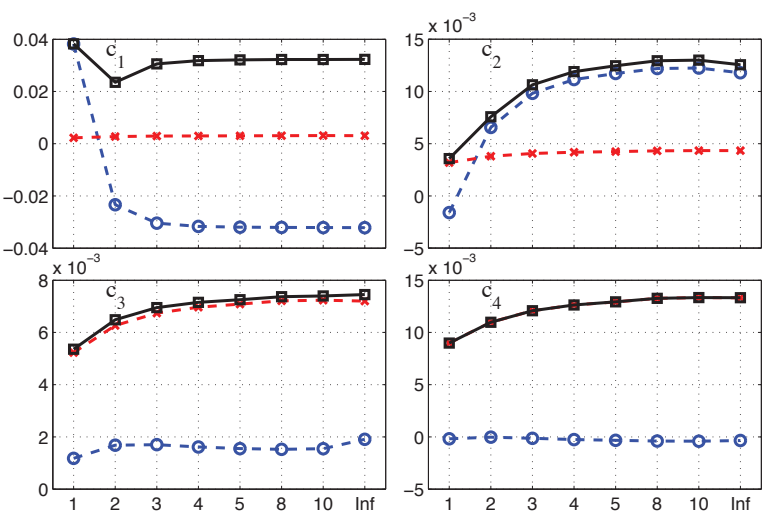

Fig. 2. Bias (blue dashed circle), standard deviation (red dashed cross) and root mean squared errors (black solid square) for the estimation of the expansion coefficients $c_{m}^{(p)}$ as functions of $p$.

\section{CONCLUSIONS}

The present contribution has shown that p-exponent and p-leader based multifractal analysis extends the classical Hölder and leader based formulation in two ways: Local regularity analysis is extended to possibly negative values, commonly observed in real world data. For processes where Hölder and p-exponents coincide, estimation performance for multifractal attributes are significantly improved compared to the reference wavelet leader formalism when tuning $p$ to small values, close to 1 . Connections between Multifractal Detrended Fluctuation Analysis, another popular tool for multifractal analysis [17] and p-leader multifractal analysis can be established and yield theoretical insights in the former tool. Also, while written explicitly for 1D signals, p-leader multifractal analysis can be extended to $\mathbb{R}^{d}$ random fields, notably for image texture analysis. These developments are under current investigations, cf. [24]. 


\section{REFERENCES}

[1] S. Jaffard, "Wavelet techniques in multifractal analysis," in Fractal Geometry and Applications: A Jubilee of Benoît Mandelbrot, M. Lapidus et M. van Frankenhuijsen Eds., Proceedings of Symposia in Pure Mathematics. 2004, vol. 72(2), pp. 91-152, AMS.

[2] R.H. Riedi, "Multifractal processes," in Theory and applications of long range dependence, P. Doukhan, G. Oppenheim, and M.S. Taqqu, Eds. 2003, pp. 625-717, Birkhäuser.

[3] H. Wendt, P. Abry, and S. Jaffard, "Bootstrap for empirical multifractal analysis," IEEE Signal Proc. Mag., vol. 24, no. 4, pp. 38-48, 2007.

[4] B. Mandelbrot, "Intermittent turbulence in self-similar cascades: divergence of high moments and dimension of the carrier," J. Fluid Mech., vol. 62, pp. 331-358, 1974.

[5] P.C. Ivanov, L.A. Nunes Amaral, A.L. Goldberger, S. Havlin, M.G. Rosenblum, Z.R. Struzik, and H.E. Stanley, "Multifractality in human heartbeat dynamics," Nature, vol. 399, pp. 461465, 1999.

[6] K. Kotani, K. Takamasu, L. Safonov, and Y. Yamamoto, "Multifractal heart rate dynamics in human cardiovascular model," Proceedings of SPIE, vol. 5110, pp. 340-347, 2003.

[7] D. Schertzer and S. Lovejoy, "Physically based rain and cloud modeling by anisotropic, multiplicative turbulent cascades," $J$. Geophys. Res., vol. 92, pp. 9693-9714, 1987.

[8] BA Toledo, AC-L Chian, EL Rempel, RA Miranda, PR Munoz, and JA Valdivia, "Wavelet-based multifractal analysis of nonlinear time series: The earthquake-driven tsunami of $27 \mathrm{febru}-$ ary 2010 in chile," Physical Review E, vol. 87, no. 2, pp. 022821, 2013.

[9] Y. Shimizu, M. Barth, C. Windischberger, E. Moser, and S. Thurner, "Wavelet-based multifractal analysis of fMRI time series.," Neuroimage, vol. 22, no. 3, pp. 1195-1202, Jul 2004.

[10] P. Ciuciu, G. Varoquaux, P. Abry, S. Sadaghiani, and A. Kleinschmidt, "Scale-free and multifractal dynamic properties of fMRI signals during rest and task," Frontiers in Physiology, vol. 3, no. 186, June 2012.

[11] L. Calvet and A. Fisher, Multifractal volatility: Theory, forecasting and pricing, Academic Press, San Diego, CA, 2008.

[12] H. Wendt, S. G. Roux, P. Abry, and S. Jaffard, "Wavelet leaders and bootstrap for multifractal analysis of images," Signal Proces., vol. 89, pp. 1100-1114, 2009.

[13] P. Abry, R. Baraniuk, P. Flandrin, R. Riedi, and D. Veitch, "Multiscale network traffic analysis, modeling, and inference using wavelets, multifractals, and cascades," IEEE Signal Processing Magazine, vol. 3, no. 19, pp. 28-46, May 2002.

[14] R. Lopes and N. Betrouni, "Fractal and multifractal analysis: A review," Medical Image analyis, vol. 13: 634-49, 2009.

[15] A. Arneodo, N. Decoster, and S.G. Roux, "A wavelet-based method for multifractal image analysis. I. Methodology and test applications on isotropic and anisotropic random rough surfaces," Eur. Phys. J. B, vol. 15, no. 3, pp. 567-600, 2000.

[16] J.F. Muzy, E. Bacry, and A. Arneodo, "The multifractal formalism revisited with wavelets," Int. J. of Bifurcation and Chaos, vol. 4, pp. 245-302, 1994.
[17] F. Soares, M. Freire, M. Pereira, F. Janela, and J. Seabra, "Towards the detection of microcalcifications on mammograms through multifractal detrended fluctuation analysis," in Proc IEEE Pacific-Rim Conf. on Multimedia, Victoria, Canada, 2009, pp. 677-681.

[18] P. Abry, S. Jaffard, and S. Roux, "Detecting oscillating singularities in multifractal analysis: Application to hydrodynamic turbulence," in Proceeding of the IEEE Int. Conf. on Acoust. Speech and Sig. Proc. (ICASSP), Praha, Cz, 2011.

[19] J. Barral and P. Gonçalves, "On the estimation of the large deviations spectrum," Journal of Statistical Physics, vol. 144, no. 6, pp. 1256-1283, 2011.

[20] E. Bacry, J. Delour, and J.F. Muzy, "Multifractal random walk," Phys. Rev. E, vol. 64: 026103, 2001.

[21] S. Jaffard and C. Melot, "Wavelet analysis of fractal boundaries," Communications In Mathematical Physics, vol. 258, no. 3, pp. 513-565, 2005.

[22] S. Mallat, A Wavelet Tour of Signal Processing, Academic Press, San Diego, CA, 1998.

[23] S. Jaffard, "Multifractal formalism for functions part ii: Selfsimilar functions," SIAM J. Math. Anal., vol. 28, no. 4, pp. 971-998, 1997.

[24] R.F. Leonarduzzi, P. Abry, S. Jaffard, S.G. Roux, M.E. Torres, and $\mathrm{H}$. Wendt, "p-exponent and p-leaders for multifractal analysis," 2014, in preparation.

[25] S. Jaffard, "Wavelet techniques for pointwise regularity," Ann. Fac. Sci. Toul., vol. 15, no. 1, pp. 3-33, 2006.

[26] S. Jaffard, P. Abry, and S.G. Roux, "Function spaces vs. scaling functions: tools for image classification," Mathematical Image processing (Springer Proceedings in Mathematics) $M$. Bergounioux ed., vol. 5, pp. 1-39, 2011.

[27] S. Jaffard, P. Abry, S. Roux, B. Vedel, and H. Wendt, The contribution of wavelets in multifractal analysis, pp. 51-98, Higher Education Press, Series in contemporary applied mathematics, China, 2010.

[28] B. Castaing, Y. Gagne, and M. Marchand, "Log-similarity for turbulent flows," Physica D, vol. 68, pp. 387-400, 1993.

[29] E. Bacry and J.F. Muzy, "Multifractal stationary random measures and multifractal random walks with log-infinitely divisible scaling laws," Phys. Rev. E, vol. 66, 2002.

[30] C.-C. Tseng, S.-C. Pei, and S.-C. Hsia, "Computation of fractional derivatives using Fourier transform and digital FIR differentiator," Signal Processing, vol. 80, no. 1, pp. 151-159, 2000. 